This is a self-archived version of an original article. This version may differ from the original in pagination and typographic details.

Author(s): Vargunin, Artjom; Silaev, Mikhail

Title: Field dependence of the vortex-core size in dirty two-band superconductors

Year: 2019

Version: Published version

Copyright: @ 2019 American Physical Society

Rights: In Copyright

Rights url: http://rightsstatements.org/page/lnC/1.0/?language=en

Please cite the original version:

Vargunin, A., \& Silaev, M. (2019). Field dependence of the vortex-core size in dirty two-band superconductors. Physical Review B, 100(1), Article 014516.

https://doi.org/10.1103/PhysRevB.100.014516 


\title{
Field dependence of the vortex-core size in dirty two-band superconductors
}

\author{
A. Vargunin \\ Department of Physics and Nanoscience Center, University of Jyväskylä, P.O. Box 35 (YFL), FI-40014 University of Jyväskylä, Finland \\ and Institute of Physics, University of Tartu, EE-50411 Tartu, Estonia \\ M. A. Silaev \\ Department of Physics and Nanoscience Center, University of Jyväskylä, P.O. Box 35 (YFL), FI-40014 University of Jyväskylä, Finland
}

(Received 29 March 2019; published 23 July 2019)

\begin{abstract}
We study the structure of Abrikosov vortices in two-band superconductors for different external magnetic fields and different parameters of the bands, including the interband scattering rate. The vortex-core size determined by the coherence lengths is found to have qualitatively different behavior from that determined by the quasiparticle density of states spatial variation. These different vortex-core length scales coincide near the upper critical field, while the discrepancy between them becomes quite significant at lower fields. Within the diffusive approximation we demonstrate several generic regimes in the field dependence of the vortex-core sizes determined by the disparity of diffusion constants in the two bands, interband pairing, and interband scattering rate.
\end{abstract}

DOI: 10.1103/PhysRevB.100.014516

\section{INTRODUCTION}

Vortex matter in multiband superconductors demonstrates many unusual properties which are drastically different from those in single-band materials [1-7]. The origin of these nontrivial new effects is the greatly enhanced number of available degrees of freedom in the system consisting of Cooper pairs and quasiparticles residing in several different bands. In this case the condensates in general tend to have different coherence lengths $[8,9]$, sharing the same critical temperature and the single divergent scale near $T_{c}$ [10]. With increasing the coupling between condensates their length scales become essentially the same [11]. Although they are quite important characteristics, the coherence lengths are not directly measurable. For example, the sizes of Abrikosov vortices in the two-band superconductor $\mathrm{MgB}_{2}$ measured with scanning tunneling microscopy (STM) local probes appear to be significantly different from the coherence length inferred from the upper critical field [4]. This physics is explained by the different localization scales of local density of states (LDOS) profiles in different bands determined by the disparity of diffusion coefficients [12]. We show that these results are strongly modified by the interband impurity scattering. The latter removes the difference between the LDOS scales in different bands and simultaneously suppresses these lengths, making them smaller than characteristic length scales of the order parameter. Interband scattering slows down the magnetic field dependence of LDOS scales.

The high-resolution of STM allows us to explore individual vortex cores in detail by measuring the quasiparticle LDOS [7, 13-16]. The LDOS profiles $N(r)$ are essentially determined by the spatial order parameter distribution $\Delta(r)$ near the vortex core. However, the vortex-core size determined from the STM tunneling conductance depends on the temperature and bias [17], indicating the spatial and energy variation of the LDOS of localized quasiparticle states trapped close to the vortex center.

The relation between zero-energy LDOS $N(r)$ and $\Delta(r)$ is quite straightforward in diffusive superconductors when the magnetic field $B$ is close to the upper critical field $H_{c 2}$ so that $H_{c 2}-B \ll H_{c 2}$. In this regime, as shown by de Gennes [18], the following relation holds:

$$
N(r)=1-2|\Delta(r)|^{2} / \Delta_{0}^{2},
$$

where $N$ is normalized to the normal-metal DOS and $\Delta_{0}$ is the gap function amplitude in the absence of magnetic field. At lower fields the relation between $N(r)$ and $\Delta(r)$ has not been checked even in the simplest case of single-band superconductors. In the present paper we demonstrate that, in general, the behaviors of these two profiles with decreasing magnetic field become quite different, so that it is not possible to extract the information about coherence length from STM measurements by directly applying Eq. (1).

The behavior of gap and LDOS profiles can be even more intriguing in two-band superconductors. According to recent experiments [7], the vortex sizes measured by STM in $2 \mathrm{H}-\mathrm{NbSe}_{2}$ and $2 \mathrm{H}-\mathrm{NbS}_{2}$ compounds demonstrate much weaker magnetic field dependencies than in the single-band materials. Interpreting these data using the de Gennes relation [18] results in the conclusion about the mostly field independent condensate length scales in two-band superconductors. Here we report the results of numerical calculations in the framework of the multiband Usadel theory. We find that in the two-band superconductor the vortex-core sizes $w_{\Delta_{1}}, w_{\Delta_{2}}$ determined by the gap function profiles in different bands $\Delta_{1,2}(r)$ in general have no distinct correlation with the widths $w_{\sigma_{1}}, w_{\sigma_{2}}$ of the corresponding LDOS distributions $N_{1,2}(r)$. We illustrate that for the distinct disparity between diffusion 
coefficients in different bands, the vortex-core sizes $w_{\sigma_{1}, \sigma_{2}}$ and $w_{\Delta_{1}, \Delta_{2}}$ can show qualitatively different behaviors as functions of the magnetic field. For large enough interband pairing the gap function distributions $\Delta_{1}(r)$ and $\Delta_{2}(r)$ are mostly identical, so that $w_{\Delta_{1}} \approx w_{\Delta_{2}}$. However, the profiles of $N_{1}(r)$ and $N_{2}(r)$ are strongly different, except for the high-field regime when the modified de Gennes relation is restored and all length scales coincide. We demonstrate that in the superconducting band with the smallest diffusion coefficient the zero-energy LDOS length scale shows quite weak magnetic field dependence. This behavior is in qualitative agreement with recent STM experiments [7] which have measured the LDOS evolution around the vortex cores in several multiband superconductors.

The structure of this paper is as follows. Our model and numerical scheme are described in Sec. II. Results are presented in Sec. III, including the single-band model (Sec. III A) and the two-band model without interband scattering (Sec. III B 1) and in the presence of interband scattering (Sec. III B 2). The work is summarized in Sec. IV.

\section{MODEL}

We use the formalism of quasiclassical Green's functions (GFs) and introduce retarded and advanced GFs, $\hat{g}_{k}=\hat{g}_{k}^{R / A}$, for a two-band $(k=1,2)$ superconductor which obey in the diffusive limit the Usadel equation

$$
D_{k} \hat{\nabla}\left(\hat{g}_{k} \cdot \hat{\nabla} \hat{g}_{k}\right)+\left[i \varepsilon \hat{\tau}_{3}+i \hat{\Delta}_{k}, \hat{g}_{k}\right]+\gamma_{k j}\left[\hat{g}_{k}, \hat{g}_{j}\right]=0
$$

for $j \neq k$. Here $D_{k}$ is the diffusion constant in each band,

$$
\hat{\Delta}_{k}=\left(\begin{array}{cc}
0 & \Delta_{k} \\
-\Delta_{k}^{*} & 0
\end{array}\right)
$$

is the gap operator, and $\hat{\nabla}=\nabla-i \pi \phi_{0}^{-1} \boldsymbol{A}\left[\hat{\tau}_{3},\right]$, where $\hat{\tau}_{3}$ is the Pauli matrix, the square brackets denote the commutator operation, and $\phi_{0}=\pi / e$ is the flux quantum. Note that we use theoretical units $k_{B}=\hbar=c=1$. The last term in Eq. (2) takes into account the interband scattering [19] determined by the amplitudes $\gamma_{k j}=\Gamma v_{j} /\left(v_{1}+v_{2}\right)$, where $\Gamma$ is the scattering rate and $v_{j}$ is the partial density of states (DOS) in the $j$ th band.

To describe the vortex structure at arbitrary fields we employ the circular-cell approximation [20-23]. Within this approach the unit cell of the hexagonal vortex lattice hosting a single vortex is replaced by a circular cell with the center at the point of superconducting phase singularity. Inside the circular cell, the gap and magnetic field distributions are taken to be radially symmetric with respect to the cell center. Below we consider the vortex state in the limit of large values of the Ginzburg-Landau parameter, $\kappa \gg 1$. In this case, magnetic field $B$ is constant inside the circular cell, and the vector potential can be taken in the form $A(r)=\varphi B r / 2$. The periodicity of the lattice solution is modeled by the special choice of the boundary conditions, namely, the vanishing supercurrent density at the circular-cell boundary. At that, the circular-cell radius is uniquely defined by magnetic induction,
$R=\sqrt{\phi_{0} /(\pi B)}$, so that there is exactly one flux quantum $\phi_{0}$ passing through the unit vortex cell.

In the $\theta$ parameterization, the GF in Nambu space reads

$$
\hat{g}_{k}=\left(\begin{array}{cc}
\cosh \theta^{(k)} & \sinh \theta^{(k)} e^{i \varphi_{k}} \\
-\sinh \theta^{(k)} e^{-i \varphi_{k}} & -\cosh \theta^{(k)}
\end{array}\right),
$$

where $\varphi_{k}$ is the band-gap phase. In cylindrical coordinates, Eq. (2) can be rewritten for complex angles $\theta^{(k)}$ as

$$
\begin{aligned}
& D_{k} r \partial_{r}\left(r \partial_{r} \theta^{(k)}\right)-D_{k}\left(1-r^{2} / R^{2}\right)^{2} \sinh \theta^{(k)} \cosh \theta^{(k)} \\
& \quad+2 i r^{2}\left(\varepsilon \sinh \theta^{(k)}-\left|\Delta_{k}\right| \cosh \theta^{(k)}\right) \\
& \quad+2 r^{2} \gamma_{k j} \sinh \left(\theta^{(j)}-\theta^{(k)}\right)=0
\end{aligned}
$$

This set of equations has to be solved self-consistently with gap order parameters determined by the conditions

$$
\left|\Delta_{k}\right|=2 \pi T \sum_{j} \lambda_{k j} \sum_{\omega_{n}>0} \sin \theta_{n}^{(j)},
$$

where $\lambda_{k j}$ are intra- and interband interaction constants which form the matrix $\hat{\lambda}, \omega_{n}=\pi T(2 n+1)$ are Matsubara frequencies, and the Matsubara GFs parametrized by $\theta_{n}^{(k)}$ satisfy (4) after the substitution $\theta^{(k)} \rightarrow-i \theta_{n}^{(k)}$ and $\varepsilon \rightarrow i \omega_{n}$. The boundary conditions read $\theta_{n}^{(k)}(r=0)=0$ and $\partial_{r} \theta_{n}^{(k)}(r=R)=0$, leading to the zero gradient of the gap modulus at the vortexcell boundary.

To find the self-consistent order parameter distributions we start by calculating the gap order parameters in the cell, taking first the initial distributions of $\left|\Delta_{1,2}(r)\right|$. By initializing guess functions $\theta_{n}^{(1,2)}$ for each $n$, we linearize the equation for the Matsubara GFs around $\theta_{n}^{(1,2)}$ and solve the linear problem numerically by applying the sweeping method. The solution provides a correction to $\theta_{n}^{(1,2)}$, and the refined guess function is used for the next iteration. By performing a sufficient number of iterations, the procedure converges to the Matsubara GFs, which are substituted into the right-hand side of Eq. (5) to obtain the correction to the initial gap functions $\left|\Delta_{1,2}\right|$. By applying refined gap functions, we repeat the scheme from the beginning and find gaps in the iterative process with the precision needed.

The zero-energy LDOS in different bands is given in the $\theta$ parametrization by $N_{k}=\cos \left(\operatorname{Im} \theta^{(k)}\right)$ at $\varepsilon=0$. To find $N_{k}$, we consider the imaginary part of Eq. (4) at zero energy with gap profiles found beforehand. We solve it numerically by starting from the guess distributions for $\theta^{(k)}$. We linearize (4) around $\theta^{(k)}$ and solve the linear problem numerically with the sweeping method. The solution gives the correction to $\theta_{k}$ which is used to construct the refined guess distribution and employ the iteration procedure.

\section{RESULTS}

\section{A. Single-band limit}

The approach presented in Sec. II reduces to the singleband model for $\lambda_{12}=\lambda_{21}=\Gamma=0$ and $D_{1,2}=D$. For $\lambda_{11}>$ $\lambda_{22}$, it corresponds to the description of the independent stronger-superconductivity band.

Figure 1 demonstrates the results of the self-consistent numerical calculations for a single-band superconductor. As magnetic field increases, the radius of the circular vortex 

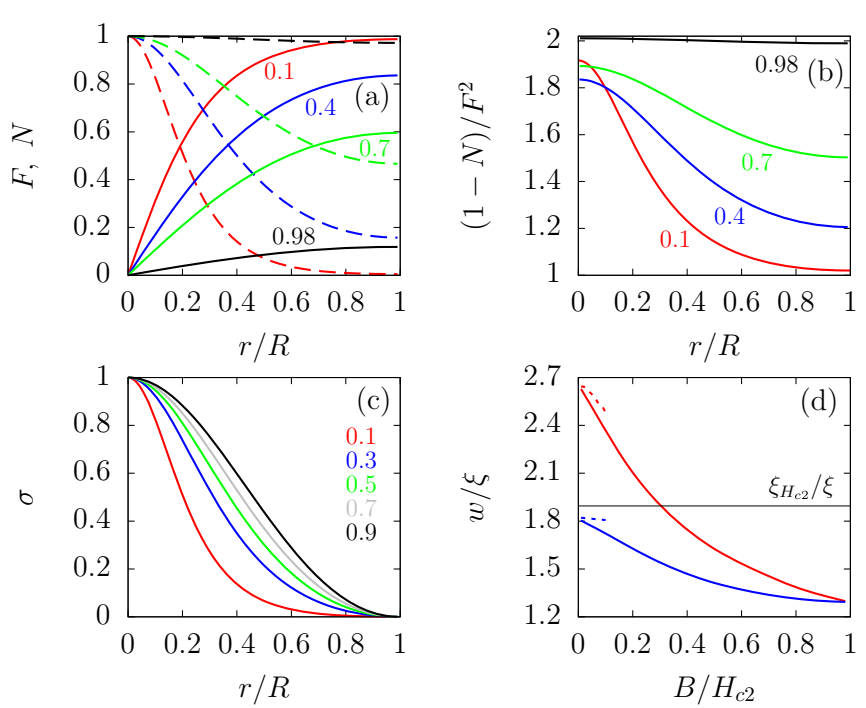

FIG. 1. Vortex structure in the single-band model at $T / T_{c}=$ 0.05 . (a) Normalized gap distribution (solid curves) inside the vortex cell, $F=|\Delta| / \Delta_{0}$. Numbers near each curve indicate the value of $B / H_{c 2}$. The dashed curves with the same color show zero-energy LDOS $N$. (b) Proportionality coefficient between $1-N$ and $F^{2}$ within the vortex cell for different magnetic fields. Note that we obtain $(1-N) / F^{2}=2$ near $H_{c 2}$, in agreement with Eq. (1). (c) LDOS variation $\sigma=\delta N(r) / \delta N(0)$ for different ratios $B / H_{c 2}$. (d) The field behavior of the vortex-core size $w=w_{\Delta}$ determined by the halfwidth of the squared gap $|\Delta|^{2}$ (red curve) and the $w=w_{\sigma}$ one defined by the half-width of the LDOS variation $\sigma$ (blue curve). Both quantities are shown in units of $\xi=\sqrt{D /\left(2 \pi T_{c}\right)}$. The dashed curves are calculated by means of Eq. (2) with substitution $\hat{\nabla} \rightarrow \nabla$ (see discussion in the text). The black horizontal line is the length scale determined by the upper critical field, $\xi_{H_{c 2}}=\sqrt{\phi_{0} /\left(2 \pi H_{c 2}\right)}$.

cell reduces, resulting in the suppression of the maximal gap value achieved at the cell boundary [see Fig. 1(a)]. Thus, the inhomogeneity of the zero-energy LDOS $N$ inside the vortex cell smooths out with rising field.

In Fig. 1(c) we plot the LDOS variation $\sigma=\delta N(r) / \delta N(0)$, where $\delta N(r)=N(r)-N(R)$, for different magnetic fields. In the single-band limit, these curves do not depend on material parameters such as the diffusion coefficient $D$ and $T_{c}$. From the definition it is clear that the LDOS variation $\sigma$ is characterized by the same half-width as the LDOS $N$ itself.

We compare the obtained LDOS and gap function profiles in order to check the range of parameters where the de Gennes relation (1) is satisfied. In principle it should be valid at low temperatures and close to $H_{c 2}$, although sometimes it is used for the analysis of the LDOS in a wider range of magnetic fields. In Fig. 1(b) we show the ratio $[1-N(r)] / F^{2}(r)$, where $F(r)=|\Delta(r)| / \Delta_{0}$, for different magnetic fields at temperature $T=0.05 T_{c}$. For high fields this ratio is constant, in agreement with Eq. (1). However, for the lower fields, $B / H_{c 2} \lesssim 0.5$, it is significantly inhomogeneous, meaning that the LDOS evolution inside the vortex cell is essentially different from the order parameter. As a result, LDOS measurements for sparse vortex lattices in general cannot be used to quantify the length scale of the superconducting order parameter.

Figure 1(d) demonstrates the field dependencies for halfwidths $w_{\Delta}$ and $w_{\sigma}$ of the squared gap $|\Delta|^{2}$ and the LDOS variation $\sigma$, respectively. Two half-widths shown in Fig. 1(d) overlap in the limit $B \rightarrow H_{c 2}$, where spatial profiles of the LDOS and $|\Delta|^{2}$ become identical [see the black curve in Fig. 1(b)]. In this case, we expect that the Abrikosov vortex lattice solution governs the behavior of the gap order parameter so that the half-width is determined by the size of the superconducting nucleus. By using a known analytic solution for the gap at $H_{c 2}$ [15] given by $F(r) \propto r e^{-r^{2} /\left(2 R^{2}\right)}$, we obtain $w_{\Delta} \approx 0.48 R$. At low temperatures, the upper critical field is determined by $e D H_{c 2} / T_{c}=\pi /\left(2 e^{C}\right)$, so that $w_{\Delta} \approx 1.3 \xi$, where $\xi^{2}=D /\left(2 \pi T_{c}\right)$, in agreement with the numerical value presented in Fig. 1(d).

For lower fields, half-widths $w_{\sigma}$ and $w_{\Delta}$ have qualitatively different behaviors, manifesting a significant difference between the squared gap and LDOS profiles and a violation of the simple relation (1). The half-width found for the squared gap coincides with previous calculations [15] and scales approximately as $w_{\Delta} \sim\left(B / H_{c 2}\right)^{-1 / 3}$ in the intermediate fields. At the same time, the half-width of the LDOS $w_{\sigma}$ changes with the field slower than that.

For very sparse vortex lattices, $B / H_{c 2} \ll 1$, both scales are characterized by linear field dependence, and for the gap we obtain $w_{\Delta}(B) / w_{\Delta}(0) \simeq 1-B / H_{c 2}$. Such behavior indicates that the spatial evolution of the gap profile is affected by the term linear in the vector potential. This conclusion can be checked by calculating vortex-core sizes in the absence of the vector potential. As a result, instead of the linear behavior we get the low-field plateaus in the dependencies of $w_{\Delta}(B)$ and $w_{\sigma}(B)$ shown by the dashed lines in Fig. 1(d). Thus, the absence of any pronounced variation of vortex-core sizes at small magnetic fields found by STM experiments [7] cannot be attributed to the specific range of magnetic field $B \ll H_{c 2}$ studied there. On the contrary, as we demonstrate below, almost-field-independent vortex-core sizes can be naturally obtained within the minimal two-band model of the superconducting state.

It is useful to compare the calculated vortex-core sizes with the experimentally measurable length scale determined by the upper critical field as $\xi_{H_{c 2}}=\sqrt{\phi_{0} / 2 \pi H_{c 2}}$. The latter can be expressed as $\xi_{H_{c 2}} \approx 1.47 w_{\Delta}\left(H_{c 2}\right)$, where in the limit $B \rightarrow H_{c 2}$ the vortex-core size is given by $w_{\Delta} \approx 0.48 R$. In the singleband superconductor the size $w_{\sigma}$ measured by STM is slightly smaller than $\xi_{H_{c 2}}$ at low fields, while the difference between these two lengths increases with magnetic field [see Fig. 1(d)]. Below we show that this picture changes qualitatively in two-band systems. We discuss the fingerprints of two-band superconductivity in STM measurements of the vortex-core sizes.

\section{B. Two-band model}

The two-band superconductivity is defined by the matrix of interaction constants $\hat{\lambda}$, the interband scattering rate $\Gamma$, and the ratio of diffusion coefficients in the bands $D_{1} / D_{2}$, which is considered below as a free parameter. For calculations, we fix intraband coupling parameters $\lambda_{11}=0.1012, \lambda_{22}=0.0448$ and consider the variable interband pairing strength.

The values of pairing constants are chosen according to the following arguments. First, the intraband pairing constants $\lambda_{11}, \lambda_{22}$ are chosen to be significantly different. Then for not 

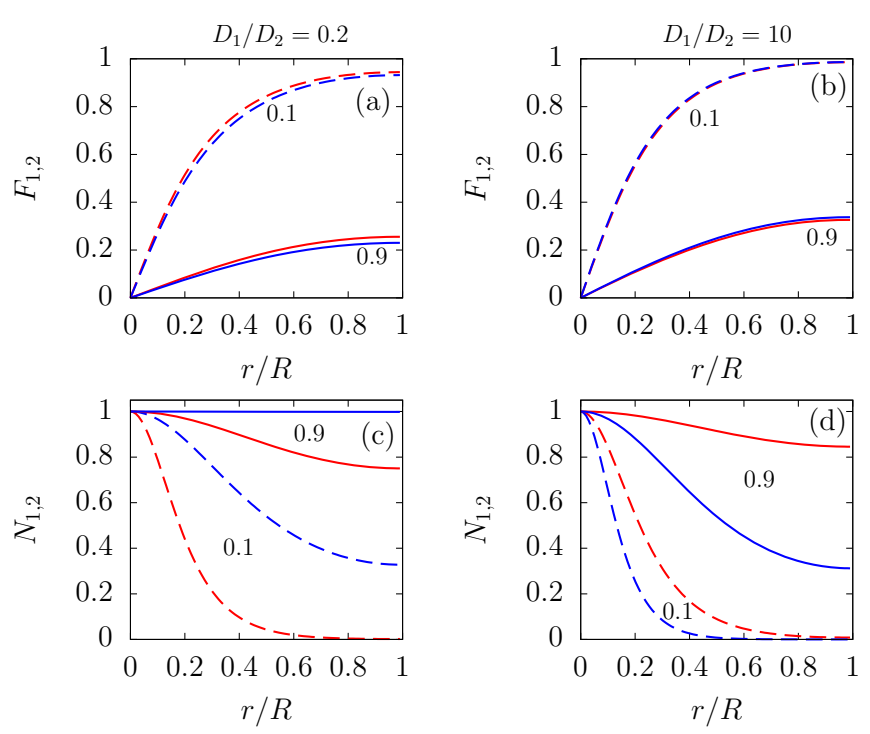

FIG. 2. Vortex structure in the two-band model at $T / T_{c}=0.1$ for $D_{1} / D_{2}=0.2,10$ (left and right columns, respectively). (a) and (b) Gap profiles in each band normalized by the bulk value, $F_{k}=$ $\left|\Delta_{k}\right| / \Delta_{k 0}$. Red and blue curves correspond to strong and weak bands $F_{1}$ and $F_{2}$, respectively. Dashed curves are for $B / H_{c 2}=0.1$, and solid curves correspond to $B / H_{c 2}=0.9$. (c) and (d) LDOS in each band $N_{k}$ for small (dashed curve) and high (solid curve) fields. Red and blue curves correspond to weak and strong bands $N_{1,2}$, respectively.

very low temperatures the pairing in the weak superconducting band, corresponding in our case to $\lambda_{22}<\lambda_{11}$, is induced by the Cooper pair tunneling from the strong band. Under such constraints the variation of $\lambda_{11}$ and $\lambda_{22}$ in wide limits does not affect the results qualitatively.

We consider several values of the interband pairing constants $\lambda_{12}, \lambda_{21}$. These parameters are not independent since their ratio is determined by the ratio of the normal-state DOS in the corresponding bands $\lambda_{12} / \lambda_{21}=\nu_{2} / \nu_{1}$. For $\mathrm{MgB}_{2}$ the interband pairing coefficients are the same order as the intraband ones [24]. To describe this case we set [25] $\lambda_{12}=$ 0.0336 and $\lambda_{21}=0.0264$. In addition, we show how vortexcore sizes change when the interband pairing is taken to be an order of magnitude smaller than for $\mathrm{MgB}_{2}, \lambda_{12}=0.00336$ and $\lambda_{21}=0.00264$.

We start with the system in the absence of interband scattering similar to the one considered in Ref. [12] but analyze the wide range of magnetic fields, changing from $B \ll H_{c 2}$ to $H_{c 2}-B \ll B$. Later, we consider the influence of the interband scattering on the vortex-core sizes.

\section{No interband scattering}

First, let us consider the system with large interband couplings $\lambda_{12}=0.0336, \lambda_{21}=0.0264$ in the absence of interband scattering $\Gamma=0$. The gap function and LDOS profiles are shown in Fig. 2 for different values of the magnetic field. The gap distributions normalized by their maximal value at the cell boundary are very close to each other independent of the values of the diffusion coefficients $D_{1,2}$. This is caused by the efficient mixing of the condensates generated by the strong interband coupling. The effect is demonstrated below explicitly by analyzing vortex-core sizes $w_{\Delta_{1,2}}$ in different bands.

In contrast to the gap profiles, LDOS distributions $N_{1,2}(r)$ are strongly affected by the band diffusion coefficients. This is seen from the Usadel equation (4), where characteristic lengths of solutions $\theta^{(1,2)}(r)$ differ by the factor $\sqrt{D_{1} / D_{2}}$. This behavior is illustrated in Figs. 2(c) and 2(d), showing that the LDOS in the band with the smaller diffusion coefficient changes at shorter distances than the one in the band with the larger diffusion coefficient.

By changing the diffusion coefficients in different bands independently, one can obtain several qualitatively different regimes. First, let us consider the case shown in Figs. 2(a) and 2(c) when the weak superconducting band has a larger diffusion coefficient. By inspecting weak-field curves, one can notice that the length scale of the LDOS $N_{2}(r)$ exceeds the scales of gap distribution $w_{\Delta_{1,2}}$. For very small magnetic fields, this regime has been studied in Ref. [12] to explain comparatively large vortex sizes measured by the STM in $\mathrm{MgB}_{2}$. By analyzing the fields ranging from zero to $H_{c 2}$, we show below that in this case the length scale of the LDOS $N_{2}(r)$ has a much stronger field dependence than other vortexcore sizes $w_{\Delta_{1,2}}$.

Another generic regime seen in Figs. 2(b) and 2(d) corresponds to the case when a band with a weaker gap has a smaller diffusion coefficient. As follows from the weak-field dependencies in Figs. 2(b) and 2(d), this results in the length scale of the LDOS $N_{2}(r)$ being much smaller than the vortexcore sizes $w_{\Delta_{1,2}}$. As we see below [Fig. 5(b), blue dashed curve], this case is characterized by significantly weak field dependence of the length scale $w_{\sigma_{2}}$.

Let us discuss the relations between the squared gap functions $\Delta_{1,2}^{2}(r)$ and LDOS deviations from the normal state, $1-N_{1,2}(r)$, shown in Fig. 3 for the two-band model. One can see that analogous to the single-band case, these profiles coincide only for the high fields close to the upper critical one. This limit can be approached analytically. For $B \approx H_{c 2}$, the order parameter is small, and it can be written as $\left|\Delta_{k}\right|=$ $c_{k} \Delta_{k 0} r e^{-r^{2} /\left(2 R^{2}\right)}$, where $c_{k}$ is a small constant (see [26]). The zero-energy solution of the spectral equation (4) is then given by $\operatorname{Im} \theta_{k}=\alpha_{k}\left|\Delta_{k}\right|$, where $\alpha_{k} e \sqrt{D_{1} D_{2}} B=-\sqrt{D_{3-k} / D_{k}}$. As a result, we obtain the relation between the LDOS and order parameter in the two-band model which is valid at fields very close to upper critical one:

$$
N_{k}=1-\frac{\left|\Delta_{k}\right|^{2}}{2 e^{2} D_{k}^{2} H_{c 2}^{2}} .
$$

This formula generalizes the de Gennes relation (1) for the multiband system and arbitrary temperatures. Indeed, in the one-band case one restores the relation (1) at low temperatures $T \rightarrow 0$ by taking into account the single-band limiting value $e D H_{c 2} / \Delta_{0}=1 / 2$. However, in the two-band case the relation between the bulk gap in a particular band and the upper critical field depends strongly on two-band model parameters, in particular, the ratio of diffusion constants. For our parameters of the two-band model and $T=0.1 T_{c}$, we have $\Delta_{10} / T_{c} \approx$ 2.05 and $\Delta_{20} / T_{c} \approx 0.81$. The upper critical field calculated using expressions from Ref. [19] reads $e H_{c 2} \sqrt{D_{1} D_{2}} / T_{c} \approx$ $1.51,0.38$ for $D_{1} / D_{2}=0.2,10$, respectively. According to 

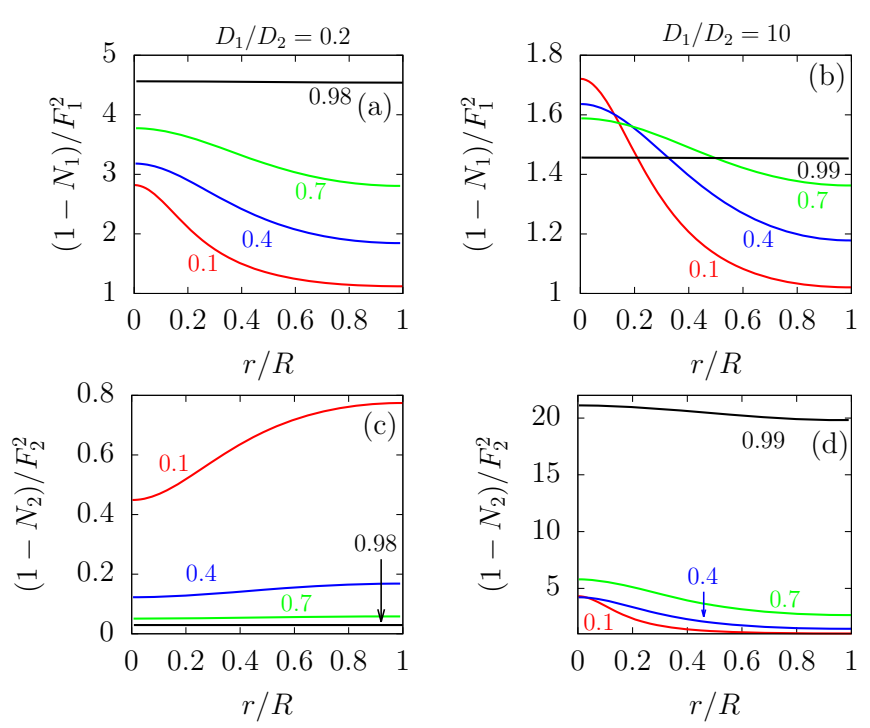

FIG. 3. Spatial variation of the proportionality coefficient between $1-N_{k}$ and $F_{k}^{2}$ in the two-band model with $D_{1} / D_{2}=0.2,10$ (left and right columns, respectively). (a) and (b) Coefficient for the stronger-superconductivity band as $B / H_{c 2}$, indicated by the colored numbers, increases. (c) and (d) Coefficient for the weakersuperconductivity band.

Eq. (6), the proportionality coefficient between $1-N_{k}$ and $\left|\Delta_{k}\right|^{2} / \Delta_{k 0}^{2}$ is then given by $4.56,1.45,0.03,23$ for the cases shown in Figs. 3(a)-3(d), respectively. These values coincide with the black curves in Fig. 3 remarkably well.

Let us consider the normalized LDOS variations within the vortex cell defined as $\sigma_{k}=\delta N_{k}(r) / \delta N_{k}(0)$, where $\delta N_{k}(r)=$ $N_{k}(r)-N_{k}(R)$, shown in Fig. 4. Unlike the single-band model, where $\sigma(r / R)$ is fixed by magnetic field [see Fig. 1(c)], in the two-band scenario there appears to be a freedom to

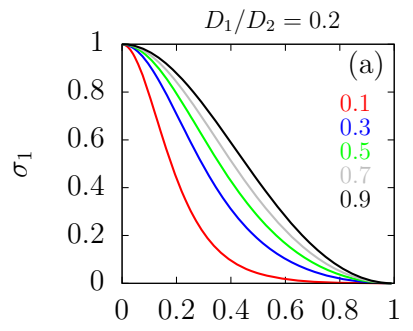

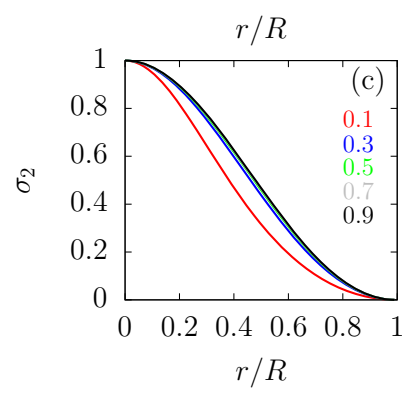
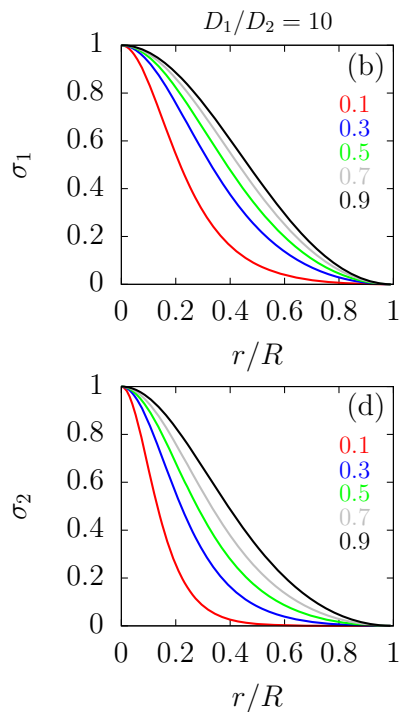

FIG. 4. LDOS variations $\sigma_{k}=\delta N_{k}(r) / \delta N_{k}(0)$ inside the vortex as the value of the normalized magnetic field $B / H_{c 2}$, indicated by different colored lines, increases. Left and right columns correspond to the cases $D_{1} / D_{2}=0.2,10$, respectively. modify LDOS variation profiles by the disparity of the band parameters. First, in the case $D_{1} \ll D_{2}$ shown in Fig. 4(c), the LDOS $N_{2}(r)$ of the band with the larger diffusion coefficient is characterized by a quite large length scale which, in fact, exceeds the vortex-cell radius $R$ already at sufficiently small magnetic fields. As a result, the corresponding LDOS and its variation $\sigma_{2}(r)$ are driven essentially by the vortex-cell radius $R$. This is reflected by the almost-field-independent form of $\sigma_{2}(r / R)$ shown in Fig. 4(c) and confirmed by the field behavior of the LDOS half-width $w_{\sigma_{2}}$ discussed below. Recent STM measurements of the multiband systems $\beta-\mathrm{Bi}_{2} \mathrm{Pd}$ [27], $2 H-\mathrm{NbSe}_{1.8} \mathrm{~S}_{0.2}$, and $2 \mathrm{H}-\mathrm{NbS}_{2}$ demonstrate very similar behaviors of the tunneling conductance distribution near the vortex core [7]. The opposite regime, $D_{1} \gg D_{2}$, illustrated in Fig. 4(d), is characterized by a sufficiently short length scale for the LDOS $N_{2}(r)$ of the band with a smaller diffusion constant. In fact, that length becomes much shorter than the vortex cell radius $R$, so that magnetic field does not significantly affect LDOS $N_{2}(r)$ profiles while changing the size of the cell. Indeed, in this case the LDOS $N_{2}$ half-width $w_{\sigma_{2}}$ is almost field independent, as we show below. Thus, by normalizing the distance by the field-dependent radius $R$, the corresponding LDOS variation $\sigma_{2}(r / R)$ profiles show quite strong modifications in magnetic field, as demonstrated in Fig. 4(d).

To quantify the above descriptive statements characterizing vortex-core sizes we calculate the lengths $w_{\Delta_{1,2}}$, determined as the half-widths of $|\Delta|_{1,2}^{2}$, and $w_{\sigma_{1,2}}$, defined as the halfwidths of $\sigma_{1,2}$. The magnetic field dependencies of these scales are shown in Fig. 5. At higher fields, all length scales approach the same value, which differs from the one obtained in the single-band limit. According to the analytical solution for the superconducting nucleus, the half-width of the squared gap at $H_{c 2}$ is given by $w_{\Delta_{k}} \approx 0.48 R$. By using values $e H_{c 2} \sqrt{D_{1} D_{2}} / T_{c}$ presented above for our model parameters, we obtain $w_{\Delta_{k}} \approx 0.97 \xi, 1.95 \xi$, where $\xi^{2}=\sqrt{D_{1} D_{2}} /\left(2 \pi T_{c}\right)$, for $D_{1} / D_{2}=0.2,10$, respectively. These values coincide with numerics presented in Figs. 5(a) and 5(b) remarkably well.

As expected, the length scales $w_{\Delta_{1,2}}$ obtained in Figs. 5(a) and $5(\mathrm{~b})$ are very close because of their almost identical spatial profiles of $F_{k}$ caused by the efficient interband pairing [see Figs. 2(a) and 2(b)]. Similar to the one-band case, length scales $w_{\Delta_{1,2}}$ can be fitted by the function $\left(B / H_{c 2}\right)^{-1 / 3}$ in a wide range of fields, $B>0.1 H_{c 2}$ [see the inset of Fig. 5(a)]. However, the characteristic length scales of the LDOS distribution $w_{\sigma_{1,2}}$ are significantly modified compared with those of the single-band system. Their field dependencies can be both stronger and weaker than that for the gap profiles determined by $w_{\Delta_{1,2}}$. In particular, in the case $D_{1} \ll D_{2}$, characterized by the leading role of the vortex-cell radius on the spatial evolution of LDOS in the band with the larger diffusion constant [see discussion of Figs. 2(c) and 4(c)], we obtain a stronger field behavior, and $w_{\sigma_{2}} \propto R \propto\left(B / H_{c 2}\right)^{-1 / 2}$ for the fields $B>0.3 H_{c 2}$ [see also the inset in Fig. 5(a)]. For weak magnetic fields, this results in the larger vortex-core size probed by STM than the correlation length determined by the upper critical field $\xi_{H_{c 2}}=\sqrt{\phi_{0} /\left(2 \pi H_{c 2}\right)}$ [12].

The opposite regime, $D_{1} \gg D_{2}$, shown in Fig. 5(b), is described by the weak field dependence of the LDOS in the band with the smaller diffusion coefficient, in agreement with 

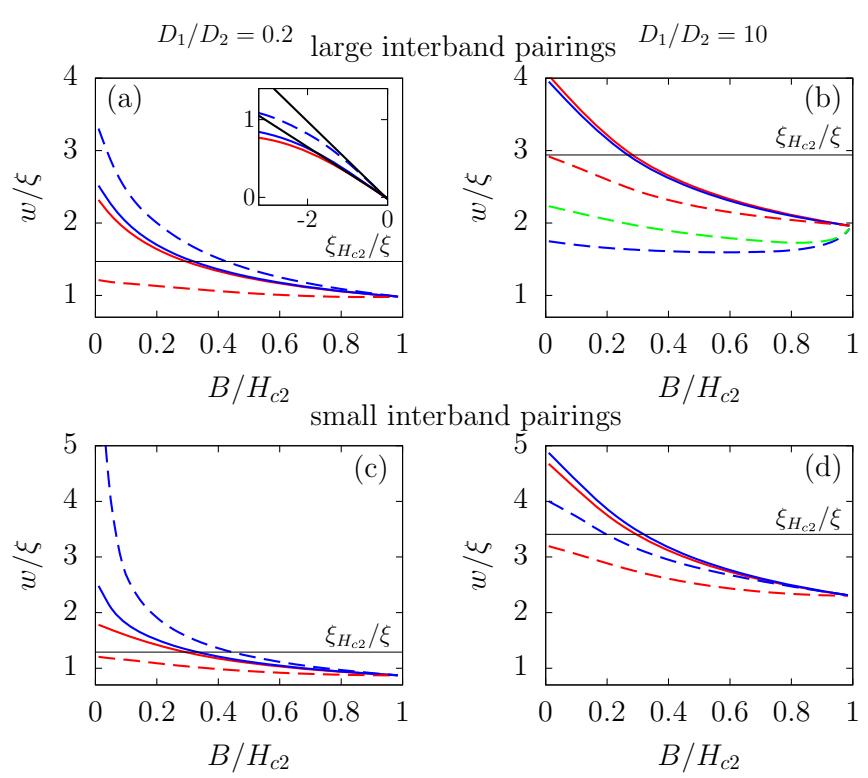

FIG. 5. Field dependence of the length scales normalized to $\xi=\sqrt{\sqrt{D_{1} D_{2}} /\left(2 \pi T_{c}\right)}$ for $D_{1} / D_{2}=0.2,10$ (left and right columns, respectively). Red and blue solid lines correspond to strong and weak band lengths $w=w_{\Delta_{1,2}}$ determined as the half-widths of $|\Delta|_{1,2}^{2}$, and red and blue dashed lines correspond to $w=w_{\sigma_{1,2}}$ defined as the half-widths of $\sigma_{1,2}$, respectively. The black horizontal line is the length scale determined by the upper critical field, $\xi_{H_{c 2}}=$ $\sqrt{\phi_{0} /\left(2 \pi H_{c 2}\right)}$. (a) and (b) Large interband pairings; (c) and (d) small interband pairings. The inset in (a) shows a logarithmic-scale plot of $w_{\Delta_{1,2}}(B)$ (red and blue solid curves) and $w_{\sigma_{2}}(B)$ (blue dashed curve). Linear dependencies $y=-x / 2+$ const (upper black curve) and $y=-x / 3+$ const (lower black curve) indicate the scalings $w_{\sigma_{2}} \sim\left(B / H_{c 2}\right)^{-1 / 2}$ and $w_{\Delta_{1,2}} \sim\left(B / H_{c 2}\right)^{-1 / 3}$ for a wide range of fields. The green dashed curve in (b) is the half-width of the total LDOS, $\left(N_{1}+N_{1}\right) / 2$.

discussions of Figs. 2(d) and 4(d). This result is in accordance with the recent STM measurements of the vortex-core sizes as a function of magnetic field [7]. Note that in principle the band-resolved LDOS distribution that we calculate is not yet the tunneling conductance measured by STM. However, the analysis of the LDOS in each of the bands is useful because it provides the understanding that in the simple model when the tunneling signal is given by the total LDOS $\left[N_{1}(r)+\right.$ $\left.N_{2}(r)\right] / 2$, the effect that we have found can significantly reduce the measured vortex size variation with magnetic field. That is, the half-width of the total LDOS shown by the dashed green curve in Fig. 5(b) has a much weaker magnetic field dependence compared to that of the single-band model demonstrated in Fig. 1(d).

Next, let us consider how the above picture changes for reduced interband pairing. In Figs. 5(c) and 5(d) we show vortex-core sizes $w_{\Delta_{k}}$ and $w_{\sigma_{k}}$ calculated for interband pairings ten times smaller than in the $\mathrm{MgB}_{2}$ case, namely, $\lambda_{12}=$ 0.00336 and $\lambda_{21}=0.00264$. For these parameters, a weakband gap is induced by the interband proximity effect and appears to be very small, $\Delta_{20} / T_{c} \approx 0.1$. According to the Usadel equation (4), this results in the enhancement of the characteristic length scale of the LDOS of the weak band, which one can observe by comparing the panels in Fig. 5 at
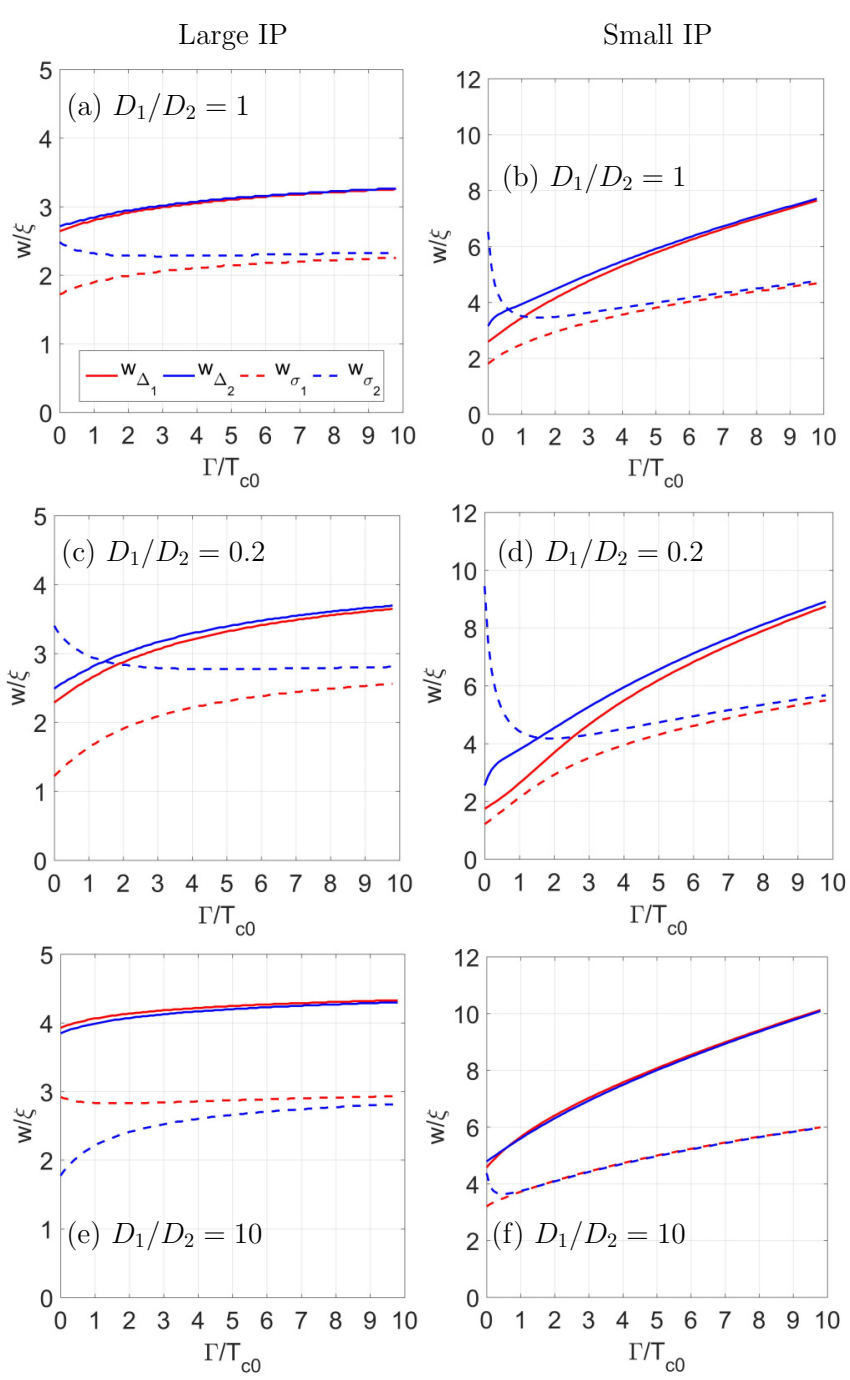

FIG. 6. Dependencies of different vortex-core sizes on the interband scattering rate for the case of (a), (c), and (e) large interband pairing (IP) $\lambda_{12}=0.0336, \lambda_{21}=0.0264$ and (b), (d), (f) small IP $\lambda_{12}=0.00336, \lambda_{21}=0.00264$ parameters as defined in the text. Different ratios of diffusion coefficients in the two bands are considered. The temperature is $T=0.1 T_{c 0}$.

weak magnetic fields. For the high fields, $B \rightarrow H_{c 2}$, we use the relation $w_{\Delta_{k}} \approx 0.48 R$ and calculate the value of the upper critical field, $e H_{c 2} \sqrt{D_{1} D_{2}} / T_{c}=1.92,0.27$, to obtain $w_{\Delta_{k}} \approx$ $0.86 \xi, 2.31 \xi$ for $D_{1} / D_{2}=0.2 ; 10$, respectively, in agreement with numerics presented in Figs. 5(c) and 5(d). Note that half-widths $w_{\sigma_{2}}$ and $w_{\Delta_{1,2}}$ shown in Fig. 5(c) have the same scaling behavior with magnetic field as in the case of large interband pairings demonstrated in the inset of Fig. 5(a).

\section{Finite interband scattering}

Here we study the effect of interband impurity scattering on the vortex-core sizes. First, we consider the region of small fields and discuss several characteristic cases corresponding to the different ratios of diffusion coefficients $D_{1} / D_{2}=0.2,1,10$ and weak/strong interband pairing, as discussed above. The dependencies of vortex-core sizes on the dimensionless scattering rate $\Gamma / T_{c 0}$ are shown in Fig. 6. Here 
$T_{c 0}$ is the critical temperature at $\Gamma=0$. In all cases the scales of order parameter distributions $w_{\Delta_{1,2}}$ are very close to each other. The scales of LDOS distributions can be quite different for small values of $\Gamma$ and $D_{2}$ significantly larger than $D_{1}$, as one can see in Figs. 6(c) and 6(d). In this regime $w_{\sigma_{2}}>w_{\sigma_{1}}$, in accordance with the results of Ref. [12] obtained for $\Gamma=0$.

Increasing the interband scattering $\Gamma$ leads to the qualitative changes in the behavior of LDOS profiles. As shown in Fig. 6, the scales of LDOS distributions $w_{\sigma_{1}}, w_{\sigma_{2}}$ get close to each other and, in general, are smaller than the sizes of vortex cores determined by the gap functions. For $D_{1} / D_{2}=0.2$ the threshold value of $\Gamma \approx 2 T_{c 0}$ determines the transition to the regime when LDOS scales are smaller than $w_{\Delta_{1,2}}$ for both large (left column) and small (right column) interband pairing parameters as defined above. For other values of the diffusion constants, e.g., $D_{1} / D_{2}=1$ and $D_{1} / D_{2}=10$, the sizes $w_{\sigma_{1,2}}$ are always smaller than $w_{\Delta_{1,2}}$, but the difference between these two types of lengths becomes more pronounced with increasing $\Gamma$. Since this feature exists for the wider range of parameters, such as the interband pairing (compare the right and left columns in Fig. 6), it can be considered the general hallmark of the multiband behavior of the vortex sizes. Quite interestingly, for small interband pairing (right column in Fig. 6) all types of vortex-core sizes grow with $\Gamma$. This is related to the decrease of the critical temperature $T_{c}(\Gamma)$ compared to $T_{c 0}$ at $\Gamma=0$. Since we fix the temperature to be $T=0.1 T_{c 0}$ in all plots, that means with increasing $\Gamma$ the temperature get closer to $T_{c}$, which causes the vortex size growth. Combined with the initial shrinkage of the LDOS scale in the weak band shown by the dashed blue curve in Figs. 6(d) and 6(f), that leads to the peculiar nonmonotonic dependence of $w_{\sigma_{2}}(\Gamma)$ shown in these plots.

Let us now consider how the shrinkage of the LDOS scale compared to the size of the gap function distribution shows up in the magnetic field dependencies of different scales. These dependencies are demonstrated in Fig. 7 for the two ratios of diffusion constants, $D_{1} / D_{2}=0.2$ (left column) and $D_{1} / D_{2}=10$ (right column). The pairing coefficients are the same as above, $\lambda_{11}=0.1012, \lambda_{22}=0.0448, \lambda_{12}=0.0336$, $\lambda_{21}=0.0264$. From Figs. 7(a) and 7(b) one can see that the main effect that we discuss in the present paper, namely, the weak dependence of the vortex-core size defined by the LDOS distribution in a band with smaller $D_{k}$, is robust against the moderate interband scattering $\Gamma / T_{c 0} \approx 1$. At the same time, the effect of the extended vortex-core size in a weak band [12] suggested for $\mathrm{MgB}_{2}$ is suppressed at $\Gamma \approx 2 T_{c 0}$ when $w_{\sigma_{2}}$ becomes smaller than vortex sizes $w_{\Delta_{k}}$. However, $w_{\sigma_{2}}$ still remains larger than $\xi_{H_{c 2}}$ until rather large values of $\Gamma$.

With a further increase of $\Gamma$ the vortex-core sizes in different bands get close to each other. Therefore, the magnetic field dependencies of both $w_{\Delta_{k}}$ and $w_{\sigma_{k}}$ are qualitatively similar to those of the single-band model. However, in contrast to the single-band case the ratios $w_{\Delta_{k}} / w_{\sigma_{k}}$ and $\xi_{H_{c 2}} / w_{\sigma_{k}}$ depend on the pairing coefficients and diffusion constants. Thus, even for large interband scatterings single- and multiband cases can be distinguished by measuring the vortex-core sizes with STM and comparing them with the scale derived from the upper critical field $\xi_{H_{c 2}}$.
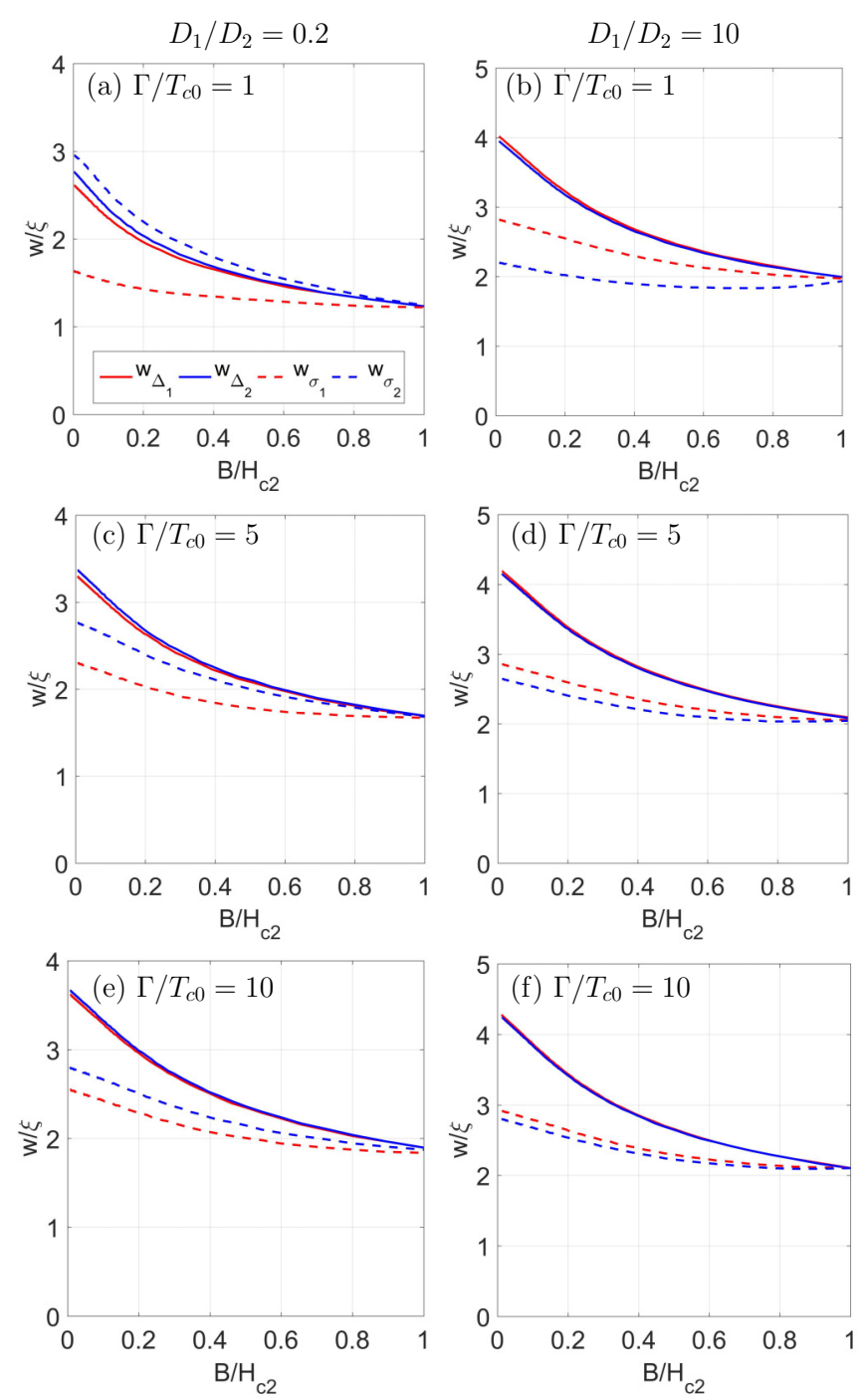

FIG. 7. Field dependencies of vortex-core sizes for different interband scattering rates and the ratios of diffusion constants (a), (c), and (e) $D_{1} / D_{2}=0.2$ and (b), (d) and (f) $D_{1} / D_{2}=10$. The interband pairing constants are $\lambda_{11}=0.1012, \lambda_{22}=0.0448, \lambda_{12}=$ $0.0336, \lambda_{21}=0.0264$. The temperature is $T=0.1 T_{c 0}$.

\section{SUMMARY}

To conclude, we demonstrated that the vortex-core size $w_{\Delta_{k}}$ determined by the healing of the gap order parameter has qualitatively different magnetic field behavior than the scale $w_{\sigma_{k}}$ defined by the spatial LDOS variations in singleand two-band dirty superconductors. We have found several generic regimes peculiar to only multiband superconductor. First, the vortex-core size $w_{\sigma_{k}}$ related to the LDOS variations in the band with the larger diffusion constant scales with the vortex-cell radius and has the field dependence stronger than the one for $w_{\Delta_{k}}$. Second, the size $w_{\sigma_{k}}$ determined by the LDOS variations in the band with the smaller diffusion constant can have significantly weaker variations with magnetic field than for $w_{\Delta_{k}}$. These features are robust against the moderate interband scattering rates. However, for stronger 
interband scattering both the LDOS distributions in different bands get very close to each other, so that the magnetic field dependencies of different vortex-core sizes are similar to that of the single-band superconductor.

\section{ACKNOWLEDGMENTS}

This work was supported by the Academy of Finland (Project No. 297439). It is our pleasure to acknowledge discussions with H. Suderow, V. Kogan, and E. Babaev.
[1] T. Nishio, V. H. Dao, Q. Chen, L. F. Chibotaru, K. Kadowaki, and V. V. Moshchalkov, Phys. Rev. B 81, 020506(R) (2010).

[2] J. Gutierrez, B. Raes, A. V. Silhanek, L. J. Li, N. D. Zhigadlo, J. Karpinski, J. Tempere, and V. V. Moshchalkov, Phys. Rev. B 85, 094511 (2012).

[3] V. Moshchalkov, M. Menghini, T. Nishio, Q. H. Chen, A. V. Silhanek, V. H. Dao, L. F. Chibotaru, N. D. Zhigadlo, and J. Karpinski, Phys. Rev. Lett. 102, 117001 (2009).

[4] M. R. Eskildsen, M. Kugler, S. Tanaka, J. Jun, S. M. Kazakov, J. Karpinski, and O. Fischer, Phys. Rev. Lett. 89, 187003 (2002).

[5] V. O. Dolocan, C. Veauvy, F. Servant, P. Lejay, K. Hasselbach, Y. Liu, and D. Mailly, Phys. Rev. Lett. 95, 097004 (2005).

[6] C. W. Hicks, J. R. Kirtley, T. M. Lippman, N. C. Koshnick, M. E. Huber, Y. Maeno, W. M. Yuhasz, M. B. Maple, and K. A. Moler, Phys. Rev. B 81, 214501 (2010).

[7] A. Fente, E. Herrera, I. Guillamón, H. Suderow, S. MañasValero, M. Galbiati, E. Coronado, and V. G. Kogan, Phys. Rev. B 94, 014517 (2016).

[8] E. Babaev, J. Carlström, and M. Speight, Phys. Rev. Lett. 105, 067003 (2010).

[9] E. Babaev and M. Speight, Phys. Rev. B 72, 180502(R) (2005).

[10] M. Silaev and E. Babaev, Phys. Rev. B 84, 094515 (2011).

[11] M. Silaev and E. Babaev, Phys. Rev. B 85, 134514 (2012).

[12] A. E. Koshelev and A. A. Golubov, Phys. Rev. Lett. 90, 177002 (2003).
[13] H. F. Hess, R. B. Robinson, and J. V. Waszczak, Phys. Rev. Lett. 64, 2711 (1990).

[14] H. F. Hess, R. B. Robinson, R. C. Dynes, J. M. Valles, and J. V. Waszczak, Phys. Rev. Lett. 62, 214 (1989).

[15] A. A. Golubov and U. Hartmann, Phys. Rev. Lett. 72, 3602 (1994).

[16] H. Suderow, I. Guillamón, J. G. Rodrigo, and S. Vieira, Supercond. Sci. Technol. 27, 063001 (2014).

[17] A. P. Volodin, A. A. Golubov, and J. Aarts, Z. Phys. B 102, 317 (1997).

[18] P. G. de Gennes, Phys. Kondens. Mater. 3, 79 (1964).

[19] A. Gurevich, Phys. Rev. B 67, 184515 (2003).

[20] D. Ihle, Phys. Status Solidi B 47, 429 (1971).

[21] R. J. Watts-Tobin, L. Kramer, and W. Pesch, J. Low Temp. Phys. 17, 71 (1974).

[22] J. Rammer, W. Pesch, and L. Kramer, Z. Phys. B 68, 49 (1987).

[23] J. Rammer, J. Low Temp. Phys. 71, 323 (1988).

[24] A. Y. Liu, I. I. Mazin, and J. Kortus, Phys. Rev. Lett. 87, 087005 (2001).

[25] V. G. Kogan, C. Martin, and R. Prozorov, Phys. Rev. B 80, 014507 (2009).

[26] A. A. Golubov and M. Y. Kupriyanov, J. Low Temp. Phys. 70, 83 (1988).

[27] E. Herrera, I. Guillamón, J. A. Galvis, A. Correa, A. Fente, R. F. Luccas, F. J. Mompean, M. García-Hernández, S. Vieira, J. P. Brison, and H. Suderow, Phys. Rev. B 92, 054507 (2015). 